\title{
ON AN ASYMPTOTIC FORMULA FOR THE MAXIMUM VOLTAGE DROP IN A ON-CHIP POWER DISTRIBUTION NETWORK
}

\author{
MARIA AGUARELES ${ }^{1}$, JAUME HARO ${ }^{2}$, JOSEP RIUS ${ }^{3}$, AND J. SOLÀ-MORALES ${ }^{2}$
}

\begin{abstract}
We present a new asymptotic formula for the maximum static voltage in a simplified model for on-chip power distribution networks of array bonded integrated circuits. In this model the voltage is the solution of a Poisson equation in an infinite planar domain whose boundary is an array of circular pads of radius $\varepsilon$, and we deal with the singular limit $\varepsilon \rightarrow 0$ case. In comparison with approximations that appear in the electronic engineering literature, our formula is more complete since we have obtained terms up to order $\varepsilon^{15}$. A procedure will be presented to compute all the successive terms, which can be interpreted as using multipole solutions of equations involving spatial derivatives of $\delta$-functions. To deduce the formula we use the method of matched asymptotic expansions. Our results are completely analytical and we make an extensive use of special functions and of the Gauss constant G.
\end{abstract}

Keywords: Asymptotic Analysis of PDE's, Weierstrass's elliptic function, Gauss constant MSC2010. Primary: 35C20. Secondary: 35J25, 35Q60.

\section{INTRODUCTION AND MAIN RESULT}

A system-on-a-chip (SOC) is an integrated circuit in which all the electronic components are included on a single chip. The design of such a device is in general complex and costly, and one has to ensure that each element obtains the right amount of power to operate efficiently. To increase the speed of operation and general performance, large voltage drops between different parts of the circuit must be avoided. In particular, it is in the design of the interconnection between the semiconductor devices and the external circuitry where special

Date: September 21, 2011.

1 IMA, UdG, Campus Montilivi, EPS-Ed. P4, E-17071 Girona, Spain, maria.aguareles@udg.edu.

2 MA1, UPC, ETSEIB, Av. Diagonal 647, E-08028 Barcelona, Spain, jaime.haro@upc.edu.

3 EE, UPC, ETSEIB, Av. Diagonal 647, E-08028 Barcelona, Spain rius@eel.upc.edu. 
care is needed. In this direction, the so-called flip-chips, with an array bonding power distribution network, represented a substantial improvement, and are actually becoming more and more used by chip designers ([15], [14]).

In Electronic Engineering one has the need for good formulas to compute the maximum voltage drop, easy to handle and ready to be used in practical tasks of chip design, even if the formulas come from simplified models. One of the simplest such models is the one that appears in the paper by Shakeri and Meindl [15], where the voltage at each point of the integrated circuit surface is modeled in terms of a solution of a Poisson equation with Dirichlet boundary values, in a domain that consists of the whole plane from which an array of circular discs of radius $\varepsilon$ has been removed, the so-called pads in the Electronic Engineering literature. This array of pads is supposed to be periodic in the two directions, with the centers of the discs separated from their neighbors by a period length $L$.

Using a combination of analytical and numerical heuristic methods, a formula was found in [15] for the maximum voltage within the SOC (minus the maximum voltage drop). That formula, in the case where $L=J_{0}=R_{s}=1$, being $R_{s}$ the sheet resistance and $J_{0}$ the consumption of current per unit area, reads

$$
\frac{1}{2 \pi} \log \varepsilon+(0.1511 \ldots)
$$

when $\varepsilon$ is small. This expression, is now widely used in the context of chip design. However, the procedure in [15] has a major drawback, and it is the fact that the method is not based on a systematic use of matched asymptotic expansions and thus cannot be easily extended to obtain further terms in the $\varepsilon$-expansion. In principle, although it is not clear from the derivation of the formula in [15], the error in this formula is order $\varepsilon$, which, at a first glance, would seem reasonable for a physical application. However, as we shall explain in Section 2 , the sizes of the pads are not that small in comparison with the size of the whole chip, which justifies the need to calculate a higher order approximation for the voltage drop.

The goal of our work has been to deduce again this formula, but now with mathematical detail in order to give some insight into the procedure behind the heuristic and limited 
method in [15]. The result that we present (see (4.4) below) reads

$$
\frac{1}{2 \pi} \log \varepsilon+\frac{1}{2 \pi} \log (\pi G)-\frac{1}{4} \varepsilon^{2}+\frac{\pi^{7} G^{8}}{300} \varepsilon^{8}+\cdots,
$$

where $G=\Gamma^{2}(1 / 4) /(2 \pi)^{3 / 2}$ (being $\Gamma$ Euler's Gamma function) is the so-called Gauss constant, which seems to be omnipresent in this problem, or, in figures,

$$
\frac{1}{2 \pi} \log \varepsilon+(0.1534 \ldots)-0.25 \varepsilon^{2}+(2.3706 \ldots) \varepsilon^{8}+\cdots .
$$

We also claim that the remaining nonzero terms will only contain powers of $\varepsilon^{8}$, and we will give a procedure to compute these successive coefficients.

To derive (1.2) we have used the techniques of the asymptotic analysis, especially in the sense of understanding the successive terms of our solution as the result of an iterative matching procedure between an inner solution, which captures the small scale effects due to the Dirichlet boundary conditions, and an outer solution that accounts for the behavior of the voltage far enough from the boundaries (see [4] or [9]). The other main ingredient has been the use of special functions and of transcendental constants. Some related works with similar problems that have also been analysed with asymptotic analysis techniques can be found in [12], [13], [16] and [17] and in the references therein.

The origin of our work is on a problem presented by researchers in Electronic Engineering in a Study Group (GEMT2009, Barcelona, see [2]), where they asked to have a mathematical look at the deduction of the formula (1.1) above, taken from [15], to see if further terms in the expansion could be obtained. At that point some preliminary answer was already given, and we built the present group of authors to study the problem more deeply.

The rest of the paper is organized as follows. In section 2 we describe the physical device, the origin and characteristics of the sytem-on-chip power distribution technology, and its relevance. The mathematical model is also justified along with the hypotheses used in its simplification. In section 3 we state precisely the mathematical problem to be considered, and find the general forms of the inner and outer solutions. As it shall be seen, we will start with a first candidate for the outer solution, namely the Green's function modified with the addition of a constant. The properties of the Green's function are stated in the form of a lemma at the end of section 3. In section 4 an iterative scheme based on an asymptotic 
matching procedure between the inner and outer solutions is presented. The next term in the expansion is computed and it is shown how to compute higher order terms. In section 5, we compare our results obtained with matching asymptotics techniques with numerically computed values of the maximum voltage drop. Section 6 is devoted to the proof of the Lemma mentioned above. Finally, the conclusions are presented in section 7 .

\section{THE MODEL}

The Power Distribution Network (PDN) of modern Integrated Circuits (ICs) is essentially composed of a grid of parallel wires in the upper conducting layer that carry the power and ground voltages from the power/ground supplies to the whole circuit [3]. In order for the IC to operate correctly, one has to ensure that the voltage drop at any point of the grid remains below a certain quantity, that is to say, the difference in the voltage between the wires carrying the power and ground should stay close enough to the power supply voltage. Thus, to reduce the voltage drop at this grid, the parallel wires should be as conductive as possible, i.e. thick and wide. The power and ground grids may be connected to the integrated circuit package in two different ways: with a so-called peripheral bonding or by means of an array bonding. The first type of connection consists of a set of supply pads which are distributed along the sides of the chip, and so the power is supplied only through the boundaries of the chip. In the second type of packaging, the so-called flip-chip PDN, the supply pads are distributed as an array over the surface of the wires of the upper conducting layer. In figure 2 there is a sketch of these two types of IC package bonding.

The PDN behaves as a conductive mesh with resistive, inductive and capacitive properties. As a consequence, the electric current spikes produced during the switching activity are transformed into voltage bounces in the supply terminals of logic cells. This bounce, which produces a reduction in the supply voltage (known as Power Supply Noise, PSN) decreases the gate drive strength, thus lowering the circuit speed performance [14]. A good PDN design must reduce the PSN below a specified value. The PSN can roughly be divided into two parts: static and dynamic. The static PSN, also called IR-drop, is due to the voltage drop produced in the PDN resistances by the average supply current, whereas the dynamic PSN is 

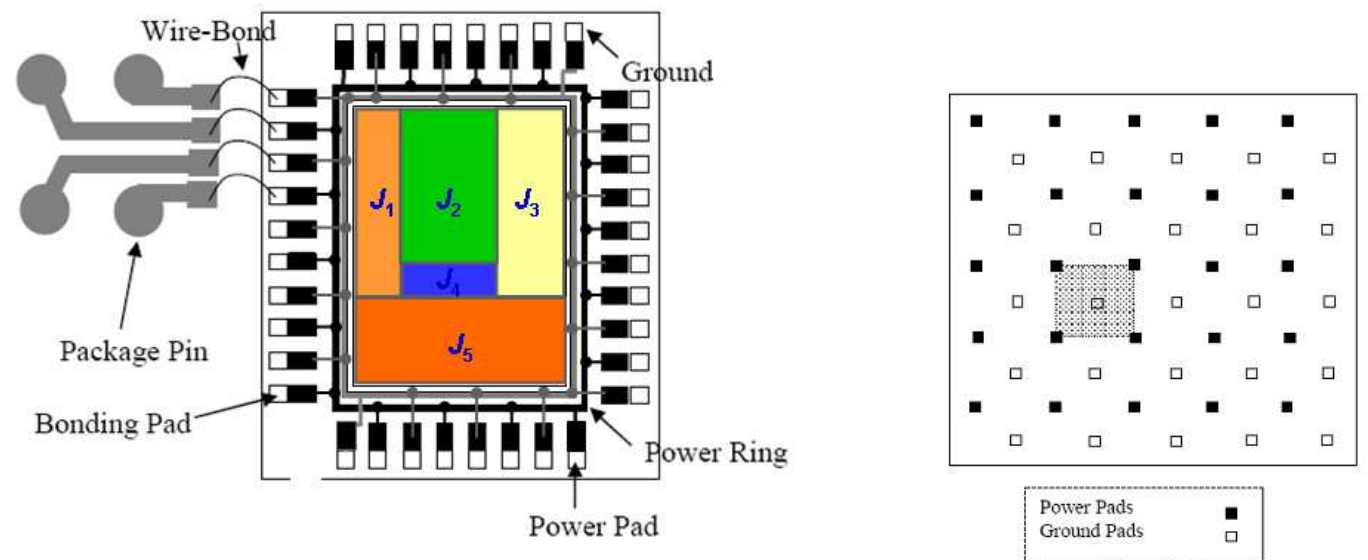

FigURE 1. Structure of a peripheral and array bonding packages.

due to the electric current transients exciting the distributed inductances and capacitances of the PDN. A precise control of the IR-drop is very important for the design of a PDN for several reasons: (i) electromigration in the PDN wires strongly depends on the average current [5]; (ii) the preliminary design of a PDN should be made according to the IR-drop specification refining the design in a later step if necessary, which is possible thanks to the fact that the average current consumption of each block in the circuit is usually estimated in advance; (iii) decoupling capacitors distributed along the IC smooth the electric current spikes in the PDN and thus the most important part of the current flowing in it is the average current [14].

The design of a good and reliable PDN is a very complex task in which designers cannot anticipate all the details. There exist widely used commercial CAD (computed-aided design) tools which are helpful but that are primarily devoted to the post-layout verification of the PDN after its complete design. This means that a failure in the design involves a costly reworking of the PDN which promotes over-dimensioning, resulting in the sacrifice of precious routing resources. These reasons necessitate of approximate pre-layout tools for the early stages of PDN design.

A typical Power/Ground grid $(\mathrm{P} / \mathrm{GG})$ is indeed a mesh of perpendicular wires strongly connected in the crossing points, horizontal wires are made with the upper metal layer 
available (free from any other routing signals) and the vertical ones are made with the second upper metal layer. A large number of vertical interconnections (vias) strongly connect the wires at the crossing points, so the whole mesh carries the power voltage level. A similar mesh carries the ground voltage. The power grid can thus be modeled as a continuous metal plate rather than a discrete grid of metal strips provided the grid is sufficiently dense, which will be our first model assumption. Therefore, in this setting the IR-drop at any point is described in terms of a Poisson equation with a source term for the current consumption, [15],

$$
\frac{1}{R_{s x}} \frac{\partial^{2} V}{\partial x^{2}}+\frac{1}{R_{s y}} \frac{\partial^{2} V}{\partial y^{2}}=J
$$

where $V$ is the voltage in $\operatorname{Volt}(\mathrm{V})$ at a point $(x, y)$ in the IC, $R_{s x}$ and $R_{s y}$ are the effective sheet resistances of the PDN in $x$ and $y$ respectively, which have units of $\mathrm{Ohm}(\Omega)$, and $J$, in Ampere per squared meter $\left(\mathrm{A} / \mathrm{m}^{2}\right)$, is the current density function at each point.

As for the boundary conditions to be imposed, they depend on the type of bonding package under consideration. In the case of a peripheral bonding the power/ground pads are connected at the four sides of the $\mathrm{P} / \mathrm{GG}$, usually using peripheral power and ground metal rings that carry the constant voltage levels. In this case one would impose constant Dirichlet boundary conditions at the four sides of the chip. In the case of an array bonding, that will be the one analyzed in this paper, the power pads are arranged in a regular mesh across the surface of the IC. This means that the actual domain will be a rectangle with a set of regularly aligned holes inside representing the pads in whose boundaries we will impose constant Dirichlet boundary conditions. As for the boundary of the whole chip, since we do not accept electric current to flow across it, we shall be imposing homogeneous Neumann boundary conditions. We note that, since the level of the power voltage is arbitrary, one can simply set it to be zero. Henceforth, we will fix $V=0$ at the boundaries of the pads which will result in the voltage being negative throughout the chip.

The pads carrying the power level are usually manufactured in rectangular shapes and they are some orders of magnitude smaller than the size of the whole domain. However, in what follows we will deal with the simpler case of circular pads which will provide explicit expressions for the maximum voltage drop that may be used as an approximation to the real 
squared-pad problem. In fact, we will discuss in the last part of this paper that the problem of square pads can be tackled in a similar same way as the circular one considered here by just performing suitable conformal transformations to map the circles into squares.

In general, ICs have a rectangular shape and their sheet resistances are not isotropic, that is to say, they differ in the $x$ and $y$ directions. However, one can always assume that the domain is an square of side one by conveniently re-scaling the $x$ and $y$ variables and considering new effective sheet resistances in both directions. For the sake of simplicity and without loss of generality we will consider in what follows that the sheet resistances are equal in both directions. The extension to an anisotropic model is straight-forward but the expressions involved turn out to be substantially more complex due to the fact that the anisotropic problem has less symmetries.

\section{The mathematical Problem}

To formulate precisely the problem, let us consider a domain $\Omega_{\varepsilon}$ consisting of the plane $(x, y)$ where we have removed the net of discs of radius $\varepsilon$ centered at the points of integer coordinates. These discs represent the pads of the chip in an idealized model. We have

$$
\Omega_{\varepsilon}=\left\{(x, y) \mid(x-k)^{2}+(y-\ell)^{2}>\varepsilon^{2}, \text { for all integers } k, \ell\right\},
$$

and we are interested in the solution $V_{\varepsilon}$ of the Poisson's problem

$$
\left\{\begin{array}{c}
\nabla^{2} V_{\varepsilon}=1 \text { in } \Omega_{\varepsilon} \\
V_{\varepsilon}(x, y)=0 \text { on } \partial \Omega_{\varepsilon} .
\end{array}\right.
$$

Because of symmetry, this solution will be periodic of period 1 in its two variables. We are interested in calculating the value of $V_{\varepsilon}$ at the points of maximum voltage, that is at the point $x=1 / 2, y=1 / 2$, or at its periodic translates.

Shakeri and Meindl obtained in [15] a good numerical approximate formula that reads

$$
V_{\varepsilon}(1 / 2,1 / 2) \simeq \frac{1}{2 \pi} \log (\varepsilon)-\frac{1}{2 \pi} \log (0.387),
$$

where the number 0.387 was obtained through a numerical process that involved a reasonable but arbitrary choice. Also the reader can observe that the formula (3.2) differs by a change 


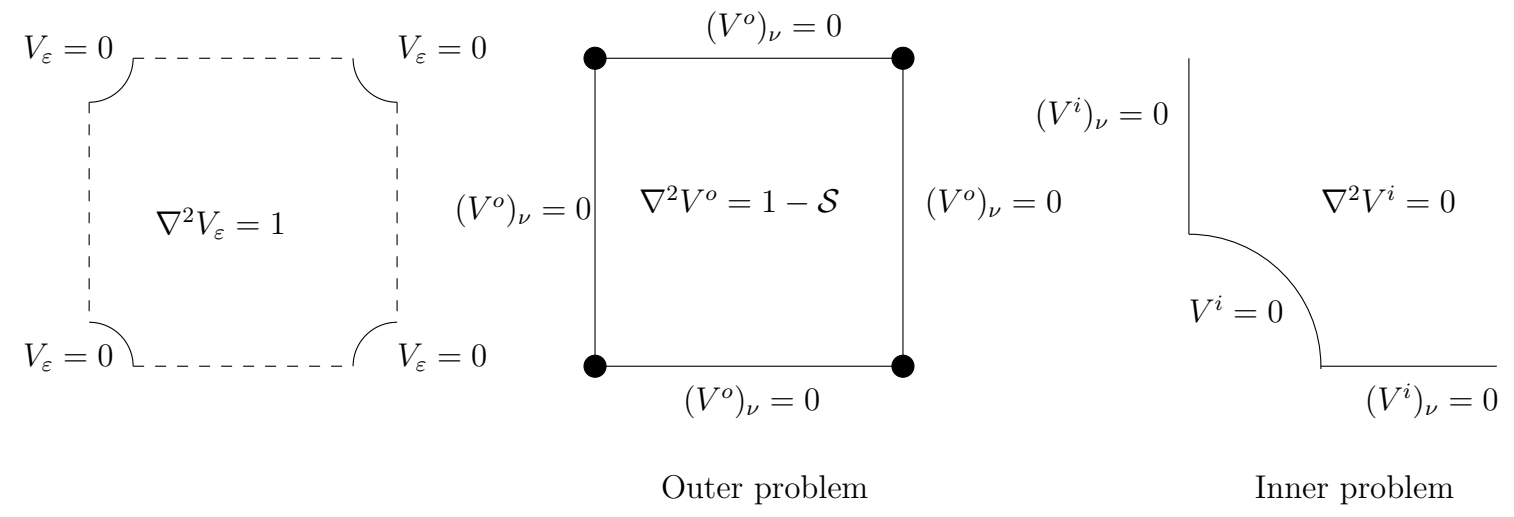

FiguRE 2. Domain under consideration, outer and inner variables domain.

of sign from the main formula (31) in [15]. This is simply due to the fact that we compute the voltage, $V$, while in their paper the formula gives the voltage drop.

In the present paper we shall present a systematic procedure to obtain the complete asymptotic expansion

$$
V_{\varepsilon}(1 / 2,1 / 2)=c_{00} \log (\varepsilon)+c_{0}+c_{1} \varepsilon+c_{2} \varepsilon^{2}+\cdots
$$

using asymptotic analysis techniques. As a result we can show that the only nonzero coefficients are $c_{00}, c_{0}, c_{2}, c_{8}, c_{16}, \ldots$ and the rest of coefficients whose indices are multiples of 8. As it was obtained in [15], $c_{00}=1 /(2 \pi)$, but with our analysis we will also be able to compute explicitly $c_{0}$ and $c_{2}$ obtaining

$$
c_{0}=\frac{1}{2 \pi} \log (\pi G) ; \quad c_{2}=-\frac{1}{4}
$$

where $G$ is the Gauss number. The other coefficients will also be analyzed, though only $c_{8}$ will be explicitly obtained. Our computation yields $c_{8}=g_{2}^{2} /(4800 \pi)=\left(\pi^{7} G^{8}\right) / 300$ being $g_{2}=4 \pi^{4} G^{4}$ the invariant of the Weierstrass's elliptic function $\wp(z)=\wp(z ; 1 / 2, i / 2)$ [1], and the remaining coefficients could also be computed in terms of this invariant.

We start by solving the boundary value problem that is represented in the first picture of Fig. 2, We have a domain $\Omega_{\varepsilon}$ that consists of the square $(0,1)^{2}$ where we have removed the four discs of radius $\varepsilon$ centered at the vertices. Our unknown function $V_{\varepsilon}$ has to satisfy 
$\nabla^{2} V_{\varepsilon}=1$ in $\Omega_{\varepsilon}$, homogeneous Dirichlet boundary conditions at the curved boundaries and homogeneous Neumann boundary conditions at the straight parts of the boundary.

To solve the problem, we use asymptotic analysis (see [4], [9]) and we break our problem into two simpler ones: the inner and the outer problem. As usual, these two solutions will turn out to contain several unknown constants, which will be determined by a matching procedure.

The outer solution $V^{o}$ has to solve the problem (3.1) but in the limit domain $\Omega_{\varepsilon}$ when $\varepsilon \rightarrow 0$. Such a domain is depicted in the second picture of Fig. 2, In $(0,1)^{2}$ it must satisfy $\nabla^{2} V^{o}=1$, homogeneous Neumann boundary conditions at the straight parts of the boundary, and a singular behavior at the corners will be required. This solution will depend on a number of arbitrary constants to be determined upon matching with the inner solution.

On the other hand, to obtain the inner solution we make the change of variables $\varepsilon x_{1}=$ $x, \varepsilon y_{1}=y$ after which the radius of the disk becomes one. In this new situation the other three disks lie at a distance of order $1 / \varepsilon$, meaning that taking formally the limit as $\varepsilon \rightarrow 0$ the domain becomes the infinite first quadrant without the disk of unitary radius centered at the origin (see the third picture in Fig. 2).

3.1. Inner region. The inner problem, in terms of the inner variables $x_{1}=x / \varepsilon, y_{1}=y / \varepsilon$, reads

$$
\left\{\begin{array}{c}
\nabla^{2} V^{i}=\varepsilon^{2} \text { in }\left\{x_{1}, y_{1}>0, x_{1}^{2}+y_{1}^{2}>1\right\} \\
V^{i}=0 \text { on } x_{1}^{2}+y_{1}^{2}=1 \\
V_{\nu}^{i}=0 \text { on } x_{1}=0 \text { or } y_{1}=0 .
\end{array}\right.
$$

A first solution to this inner problem is readily found to be

$$
V_{1}^{i}\left(x_{1}, y_{1}\right)=\alpha_{00} \log \left|z_{1}\right|-\frac{\varepsilon^{2}}{4}+\frac{\varepsilon^{2}}{4}\left|z_{1}\right|^{2}
$$

where $z_{1}=x_{1}+i y_{1}$ and $\alpha_{00}$ is unknown, but this expression does not exhaust all the possible solutions. With the method of separation of variables we see that we can add to (3.5) any linear combination of functions of the form $\mathfrak{R e}\left(z_{1}^{n}-z_{1}^{-n}\right)$. To satisfy the boundary condition at $x_{1}=0$ we have to require $n \geq 1$ to be an even number, although, as we shall see later on, $n$ will in fact be restricted to be a multiple of 4 . 
Recall that the potentials of the form $\mathfrak{R e}\left(z_{1}^{-n}\right)$ are called multipoles in the classical electromagnetics literature (see [10], e.g.).

With these ideas in mind we then pose the following solution for the inner equation,

$$
V^{i}\left(x_{1}, y_{1}\right)=\alpha_{00} \log \left|z_{1}\right|-\frac{\varepsilon^{2}}{4}+\frac{\varepsilon^{2}}{4}\left|z_{1}\right|^{2}+\sum_{n \geq 1} \alpha_{n} \varepsilon^{n} \mathfrak{R e}\left(z_{1}^{n}-z_{1}^{-n}\right),
$$

where the coefficients $\alpha_{n}$ will be determined by matching with the outer solution.

3.1.1. Outer limit of the inner. Upon inspecting expression (3.6) one realises that the leading order behaviour as $z_{1} \rightarrow \infty$ is governed by a logarithmic term followed by an order one magnitude. We must then find solutions of the outer problem yielding this kind of behaviour as $z \rightarrow 0$.

3.2. Outer region. The problem to be solved in the outer region is then

$$
\left\{\begin{array}{c}
\nabla^{2} V^{o}=1-\mathcal{S} \text { in }(0,1)^{2} \\
\left(V^{o}\right)_{\nu}=0 \text { on } \partial\left\{(0,1)^{2}\right\}
\end{array}\right.
$$

where $\mathcal{S}$ denotes a set of singularities at the vertices of the domain. The outer solution is only valid away from such singular points, but one must bear in mind that the type of solutions that will match with the inner must be singular at the corners of the domain. Furthermore, such singular terms are needed in order to obtain a non-trivial solution. We anticipate that one of the difficulties in this problem is to define the right type of singularity that gives place to a solution that matches with the inner.

To solve (3.7), following [13], [17], we pose the following expansion in powers of $\varepsilon$,

$$
V^{o} \sim V_{0}^{o} \log \varepsilon+V_{1}^{o}+\varepsilon V_{2}^{o}+\ldots
$$

and we also express the singular term as a sum, in powers of $\varepsilon$, of a set of singularities like,

$$
\mathcal{S} \sim \mathcal{S}_{1}+\varepsilon \mathcal{S}_{2}+\ldots
$$

At this point we must clarify that this last expression is just an abuse of notation to emphasize the fact that each term $V_{k}^{o}$ will need to have a very specific singular behaviour at the corners of the square in order to match with the inner. 
One trivially finds that $V_{0}^{o}=C_{0}$, being $C_{0}$ an unknown constant, $V_{1}^{o}$ satisfies

$$
\left\{\begin{array}{l}
\nabla^{2} V_{1}^{o}=1-\mathcal{S}_{1} \text { in }(0,1)^{2} \\
\left(V_{1}^{o}\right)_{\nu}=0 \text { on } \partial\left\{(0,1)^{2}\right\}
\end{array}\right.
$$

and the rest of terms, $V_{k}^{o}$ for $k=2,3, \ldots$ will be found as solutions to

$$
\left\{\begin{array}{c}
\nabla^{2} V_{k}^{o}=-\mathcal{S}_{k} \text { in }(0,1)^{2} \\
\left(V_{k}^{o}\right)_{\nu}=0 \text { on } \partial\left\{(0,1)^{2}\right\}
\end{array}\right.
$$

We then start by solving (3.8) and we take, for the singular part,

$$
\mathcal{S}_{1}=\left(\delta_{(0,0)}+\delta_{(0,1)}+\delta_{(1,1)}+\delta_{(1,0)}\right)
$$

This choice of $\mathcal{S}_{1}$ is not arbitrary, on the contrary, it provides a singular behaviour at the corners but it does also satisfy the zero mean-value condition that the right hand side in (3.8) must satisfy,

$$
\int_{(0,1)^{2}} S_{1}=1
$$

Therefore, $V_{1}^{o}$ represents the doubly-periodic Green's function of $\nabla^{2}$ in the plane. We solve problem (3.8) with the method of double cosine series:

$1-\delta_{(0,0)}-\delta_{(0,1)}-\delta_{(1,1)}-\delta_{(1,0)}=-\sum_{\ell=1}^{\infty} 2(\cos (2 \pi \ell x)+\cos (2 \pi \ell y))-\sum_{n, m=1}^{\infty} 4 \cos (2 \pi n x) \cos (2 \pi m y)$ and then $V_{1}^{o}=C_{1}+v_{1}+v_{2}$, where $C_{1}$ is again an unknown constant to be determined by matching with the inner,

$$
v_{1}(x, y)=\sum_{\ell=1}^{\infty} \frac{1}{2 \pi^{2} \ell^{2}}(\cos (2 \ell \pi x)+\cos (2 \ell \pi y))
$$

and

$$
v_{2}(x, y)=\sum_{n, m=1}^{\infty} \frac{1}{\pi^{2}\left(n^{2}+m^{2}\right)} \cos (2 n \pi x) \cos (2 m \pi y) .
$$

The next two terms, $V_{2}^{o}$ and $V_{3}^{o}$, as it will become clear in Section 4 when we match, are going to be just zero and an unknown constant respectively, $V_{2}^{o}=0, V_{3}^{o}=C_{3}$. The following terms, $V_{k}^{o}$ with $k$ ranging from four to eight, will be found to be zero, as it shall be justified later on. 
3.2.1. Inner limit of the outer. We now inspect the leading order outer solution $V_{1}^{o}(x, y)$ and analyse it locally, near $x=y=0$. The general form of a function $u(x, y)$ that satisfies $\nabla^{2} u=1$ in a neighborhood of $(0,0)$ but that is allowed to be singular at $(0,0)$ is

$$
u(x, y)=\frac{1}{4}|z|^{2}+\beta_{00} \log |z|+\mathfrak{R e}\left(\beta_{0}+\sum_{n>0} \beta_{n} z^{n}+\sum_{n<0} \beta_{n} z^{n}\right)
$$

where $z=x+i y$. This just comes from a Laurent series and the terms of the singular part (the last sum) correspond to the multipoles. The coefficients $\beta_{n}$ are, in principle, complex numbers.

If we impose now the local symmetries of the problem, the solution $u$ has to be also invariant under the changes $x \leftrightarrow-x, y \leftrightarrow-y$ and $x \leftrightarrow y$ (the invariance with respect to the first change can in fact be deduced from the invariance with respect to the other two). Applied to a monomial $\mathfrak{R e}\left(\beta_{n} z^{n}\right)$ the symmetry $z \leftrightarrow-z$ implies that $n$ has to be an even number. The symmetry $z \leftrightarrow \bar{z}$ implies that the coefficients $\beta_{n}$ must be real. Finally, the symmetry $x \leftrightarrow y$, or, equivalently, $z \leftrightarrow e^{-i \pi / 4} \overline{e^{i \pi / 4} z}$ implies that $n$ has to be a multiple of 4 . So we have that

$$
u(x, y)=\beta_{00} \log |z|+\beta_{0}+\frac{1}{4}|z|^{2}+\sum_{n>0} \beta_{4 n} \mathfrak{R e}\left(z^{4 n}\right)+\sum_{n<0} \beta_{4 n} \mathfrak{R e}\left(z^{4 n}\right),
$$

where all the coefficients $\gamma_{4 n}$ are fixed and real and $C_{1}$ is an arbitrary constant.

We now need a power series approximation in $\varepsilon$ for $v_{1}(x, y)+v_{2}(x, y)$ near $x=y=0$, which we will show to be of the shape predicted in (3.13) Also, in order to compute the maximum voltage, we will need to evaluate $v_{1}(1 / 2,1 / 2)+v_{2}(1 / 2,1 / 2)$. These are longer calculations, and the results are summarized in the following statement, whose proof we postpone until section 6 below.

\section{Lemma.}

$$
\left(v_{1}+v_{2}\right)(1 / 2,1 / 2)=-\frac{\log 2}{4 \pi}
$$

and also

$$
\left(v_{1}+v_{2}\right)(x, y)=\gamma_{00} \log |z|+\gamma_{0}+\gamma_{2}|z|^{2}+\sum_{n=1}^{\infty} \gamma_{4 n} \mathfrak{R e}\left(z^{4 n}\right)
$$


with

$$
\gamma_{00}=\frac{-1}{2 \pi}, \quad \gamma_{0}=\frac{1}{2 \pi} \log \left(\frac{\sqrt{2}}{2 \pi G}\right)
$$

where $G$ is the Gauss constant, $\gamma_{2}=\frac{1}{4}$, and the coefficients $\gamma_{4 n}$ are related with the invariant $g_{2}$ (which we will show to be $g_{2}=4 \pi^{4} G^{4}$ ) of the Weierstrass's elliptic function $\wp(z) \equiv$ $\wp(z ; 1 / 2, i / 2)$ through the formulae

$$
\gamma_{4 n}=\frac{1}{8 n \pi} G_{4 n}
$$

being $G_{4 n}=\sum_{+m \neq 0} \frac{1}{(m+i n)^{4 n}}$ the Einsestein series that satisfy $G_{4}=g_{2} / 60$ and $G_{4 n}=$ $\frac{3}{\left(16 n^{2}-1\right)(2 n-3)} \sum_{m=1}^{n-1}(4 m-1)(4(n-m)-1) G_{4 m} G_{4(n-m)}$.

Therefore, the inner limit of the leading order outer reads,

$$
V_{1}^{o}\left(x_{1}, y_{1}\right) \sim C_{1}-\frac{1}{2 \pi} \log |z|+\frac{1}{2 \pi} \log \left(\frac{\sqrt{2}}{2 \pi G}\right)+\frac{1}{4}|z|^{2}+\sum_{n=1}^{\infty} \gamma_{4 n} \mathfrak{R e}\left(z^{4 n}\right) .
$$

\section{The iterative MATChing PROCESs AND the FinAl FORMUla}

4.1. Leading orders matching. Once the statement of this lemma is accepted, one can easily match the two-term inner expansion of the outer to the three-term outer expansion of the inner (in the notation of Van Dyke [18] we impose $(2 \mathrm{ti})(3 \mathrm{to})=(3 \mathrm{to})(2 \mathrm{ti}))$. Writting $V_{o}$ and $V_{i}$ both in terms of the inner coordinates $z_{1}=z / \varepsilon$, gives

$$
\begin{gathered}
V_{0} \sim C_{0} \log \varepsilon-\frac{1}{2 \pi} \log \left(\varepsilon\left|z_{1}\right|\right)+C_{1}+\frac{1}{2 \pi} \log \left(\frac{\sqrt{2}}{2 \pi G}\right)+\frac{1}{4} \varepsilon^{2}\left|z_{1}\right|^{2}+C_{3} \varepsilon^{2}+O\left(\varepsilon^{3}\right), \\
V_{i} \sim \alpha_{00} \log \left|z_{1}\right|-\frac{\varepsilon^{2}}{4}+\frac{\varepsilon^{2}}{4}\left|z_{1}\right|^{2}+\varepsilon \alpha_{1} \mathfrak{R e}\left(z_{1}-1 / z_{1}\right)+\varepsilon^{2} \alpha_{2} \mathfrak{R e}\left(z_{1}^{2}-1 / z_{1}^{2}\right)+O\left(\varepsilon^{3}\right),
\end{gathered}
$$

from where it is readily found that $C_{0}=1 /(2 \pi), \alpha_{00}=-1 /(2 \pi), \alpha_{1}=\alpha_{2}=0, C_{1}=$ $-1 /(2 \pi) \log (\sqrt{2} /(2 \pi G))$ and $C_{3}=-1 / 4$.

4.2. Computation and matching of higher order terms. At this point we have a first approximation to the outer solution that matches with $V^{i}$ only up to some terms. More precisely, the error has a dominant term of order of $\varepsilon^{4}$ (in the inner coordinates), given by

$$
\varepsilon^{4} \gamma_{4} \mathfrak{R e} z_{1}^{4}=\gamma_{4} \mathfrak{R e} z^{4}
$$


since it would cause $\alpha_{4}=\gamma_{4}$ and hence would cause a term of the form $\gamma_{4} \varepsilon^{8} \mathfrak{R e} z_{1}^{-4}$ that would not match with the outer. It hence seems that one must choose $V_{k}^{o}=0$ for $k=4 . .8$ and consider the term $V_{9}^{o}$ as a candidate to produce a term that balances with $\gamma_{4} \varepsilon^{8} \mathfrak{R e} z_{1}^{-4}$.

We note that we already have the whole inner expansion, which helps to determine the type of singular terms that we will need to consider in the outer problem. Thence, it is obvious that expression $\gamma_{4} \mathfrak{R e} z^{4}$ would match with (3.6) if we added the correction term $-\gamma_{4} \varepsilon^{8} \mathfrak{R e} z^{-4}$ and we took $\alpha_{4}=\gamma_{4}$. However, the simple choice of taking $V_{9}^{o}=-\gamma_{4} \mathfrak{R e} z^{-4}$ would not be good enough, since it cancels the right error term and it is symmetric under $x \leftrightarrow-x, y \leftrightarrow-y$ and $x \leftrightarrow y$ but it is not symmetric under $x \leftrightarrow(1-x)$ and $y \leftrightarrow(1-y)$. In other words, we have to modify or extend the multipole $\gamma_{4} \varepsilon^{8} \mathfrak{R e} z^{-4}$ solution to satisfy the boundary conditions that have been considered in (3.7).

By using distribution theory, it is not difficult to see that the multipole function $u=\mathfrak{R e} z^{-4}$, which, for $(x, y) \neq(0,0)$ coincides with $(1 / 6) D_{x}^{2} D_{y}^{2} \log r$, satisfies, in the whole $\mathbb{R}^{2}$,

$$
\nabla^{2} u=-\frac{2 \pi}{3} \mathfrak{R e}\left(D_{z}^{4}\right) \delta_{(0,0)}=-\frac{\pi}{24}\left(D_{x}^{4}-6 D_{x}^{2} D_{y}^{2}+D_{y}^{4}\right) \delta_{(0,0)}
$$

where the derivatives $D_{x}, D_{y}$ and $D_{z}$ are understood in the sense of distributions. Thus, the idea is to compute $V_{9}^{o}$ as a solution of

$$
\left\{\begin{array}{c}
\nabla^{2} V_{9}^{o}=\mathcal{S}_{9} \text { in }(0,1)^{2} \\
\left(V_{9}^{o}\right)_{\nu}=0 \text { on } \partial\left\{(0,1)^{2}\right\}
\end{array}\right.
$$

with

$$
\mathcal{S}_{9}=\gamma_{4} \frac{\pi}{24}\left(D_{x}^{4}-6 D_{x}^{2} D_{y}^{2}+D_{y}^{4}\right)\left(\delta_{(0,0)}+\delta_{(0,1)}+\delta_{(1,1)}+\delta_{(1,0)}\right)
$$

Such solution, $V_{9}^{o}$, will behave like $-\gamma_{4} \mathfrak{R e} z^{-4}$ locally close to $x=y=0$, but will also satisfy all the symmetry requirements.

In fact, inspired by the definition of the Weierstrass' elliptic function of $\wp(z)=\wp(z ; 1 / 2, i / 2)$, we have chosen

$$
V_{9}^{o}(x, y)=-\gamma_{4} \mathfrak{R e}\left[\frac{1}{z^{4}}\right]_{p e r}
$$


being

$$
\begin{gathered}
{\left[\frac{1}{z^{4}}\right]_{p e r} \equiv \frac{1}{z^{4}}+\sum_{(m, n) \neq(0,0)}\left[\frac{1}{(z-(m+i n))^{4}}-\frac{1}{(m+i n)^{4}}\right]} \\
=\sum_{(m, n)} \frac{1}{(z-(m+i n))^{4}}-\frac{\pi^{4} G^{4}}{15}
\end{gathered}
$$

which is a solution to problem (4.1).

However, we are only interested in calculating the value of $V_{9}^{o}(1 / 2,1 / 2)$ which is related with the invariant $g_{2}$ through the formula $V_{9}^{o}(1 / 2,1 / 2)=\gamma_{4} g_{2} / 10=g_{2}^{2} /(4800 \pi)$. Indeed, from the definitions of $\wp(z)$ and $\left[1 / z^{4}\right]_{p e r}$, one obtains the relation $\wp^{\prime \prime}(z)=6\left[1 / z^{4}\right]_{p e r}+g_{2} / 10$ which means that

$$
V_{9}^{o}(x, y)=-\frac{\gamma_{4}}{6} \mathfrak{R e}\left(\wp^{\prime \prime}(z)-g_{2} / 10\right) .
$$

Now we use formula (8.1.6) in [1] $\left(\wp^{\prime}(z)\right)^{2}=4 \wp^{3}(z)-g_{2} \wp(z)$, and taking twice the derivative of this expression and evaluating at $z=1 / 2+i / 2$ one obtains $\wp^{\prime \prime}(1 / 2+i / 2)=-g_{2} / 2$, since $\wp(1 / 2+i / 2)=\wp^{\prime}(1 / 2+i / 2)=0$, which proves our statement.

Let us now obtain the final formula by merging the results we have obtained so far. The value we are looking for is the voltage at the point $(1 / 2,1 / 2)$, so we have to compute the following:

$$
\begin{aligned}
V^{o}(1 / 2,1 / 2) & \sim V_{0}^{o}(1 / 2,1 / 2) \log \varepsilon+V_{1}^{o}(1 / 2,1 / 2)+V_{3}^{o}(1 / 2,1 / 2) \varepsilon^{2}+V_{9}^{o}(1 / 2,1 / 2) \varepsilon^{8}+\cdots \\
& \sim \frac{1}{2 \pi} \log \varepsilon+\frac{1}{2 \pi} \log (\pi G)-\frac{1}{4} \varepsilon^{2}+\frac{\pi^{7} G^{8}}{300} \varepsilon^{8}+\cdots
\end{aligned}
$$

that is our final formula (compare with (3.2)).

The next term in the expansion. Following the same asymptotic scheme one can show that the next non-zero term in the outer expansion would be $V_{17}^{o}$ with which one would match up to order $\varepsilon^{16}$, while $V_{25}^{o}$ would give correction terms up to $\varepsilon^{24}$, and so on. Indeed, taking into account the relation between $\wp(z)$ and $\left[\frac{1}{z^{4}}\right]_{\text {per }}$ one can write

$$
\begin{aligned}
V^{o}(x, y) & \sim-\frac{1}{2 \pi} \log |z| \varepsilon \mid+\frac{1}{4}\left(|z|^{2}-\varepsilon^{2}\right)+\gamma_{4}\left(\mathfrak{R e} z^{4}-\varepsilon^{8} \mathfrak{R e} z^{-4}\right) \\
& -35 \gamma_{4} G_{8} \varepsilon^{8} \mathfrak{R e} z^{4}+\sum_{n=2}^{\infty}\left(\gamma_{4 n}-\varepsilon^{8} \gamma_{4} \frac{(2 n+1)(4 n+1)(4 n+3)}{3} G_{4 n+4}\right) \mathfrak{R e} z^{4 n},
\end{aligned}
$$


which shows that the terms causing now misbalance are

$$
-35 \gamma_{4} G_{8} \varepsilon^{12} \mathfrak{R e} z_{1}^{4}+\gamma_{8} \varepsilon^{8} \mathfrak{R e} z_{1}^{8}
$$

So then, to match this expression with (3.6) one must choose

$$
V_{17}^{o}(x, y)=35 \gamma_{4} G_{8} \mathfrak{R e}\left[\frac{1}{z^{4}}\right]_{p e r}-\gamma_{8} \mathfrak{R e}\left[\frac{1}{z^{8}}\right]_{p e r},
$$

being

$$
\left[\frac{1}{z^{8}}\right]_{p e r} \equiv \frac{1}{z^{8}}+\sum_{(m, n) \neq(0,0)}\left[\frac{1}{(z-(m+i n))^{8}}-\frac{1}{(m+i n)^{8}}\right]=\sum_{(m, n)} \frac{1}{(z-(m+i n))^{8}}-\frac{\pi^{8} G^{8}}{525}
$$

which effectively gives a correction of order $\varepsilon^{16}$.

\section{Comparison With NUMerical Simulations}

To compare our solution (4.4) for the maximum value of the voltage drop with numerical simulations we have solved equation (3.1) taking into account the symmetries of the problem by solving on $\Omega_{\epsilon}$ one eight of the square with one eight of the circular pad. We have then used the Finite Elements Method with a Galerkin scheme, with a parallel sparse direct linear solver to solve the linear system of equations for the values of the solutions at the nodes of the mesh. In particular we have used the Finite Elements Method solver software COMSOL Multiphysics and we have meshed the domain with bilinear elements.

The results, which have been plotted in figure 3, show the accuracy of our formula (4.4) even for quite large values of the radius. In particular, the figure at the right hand side shows that formula (4.4) provides substantially more accurate values when the radius of the pad becomes larger than 0.3 .

Finally, it has been also numerically checked that the next term in the expansion is indeed of order $\epsilon^{16}$. This is seen by plotting the difference between formula (4.4) and the numerical value of the voltage drop against the radius $\epsilon$ in a logarithmic scale. If such difference is indeed of order $\epsilon^{16}$ it should be fitted into a straight line of slope 16. Figure 4 shows precisely that the difference has indeed a linear fit which is found to be given by the equation $15.98 \log \varepsilon+5.823$ with a confidence bound of $95 \%$. 

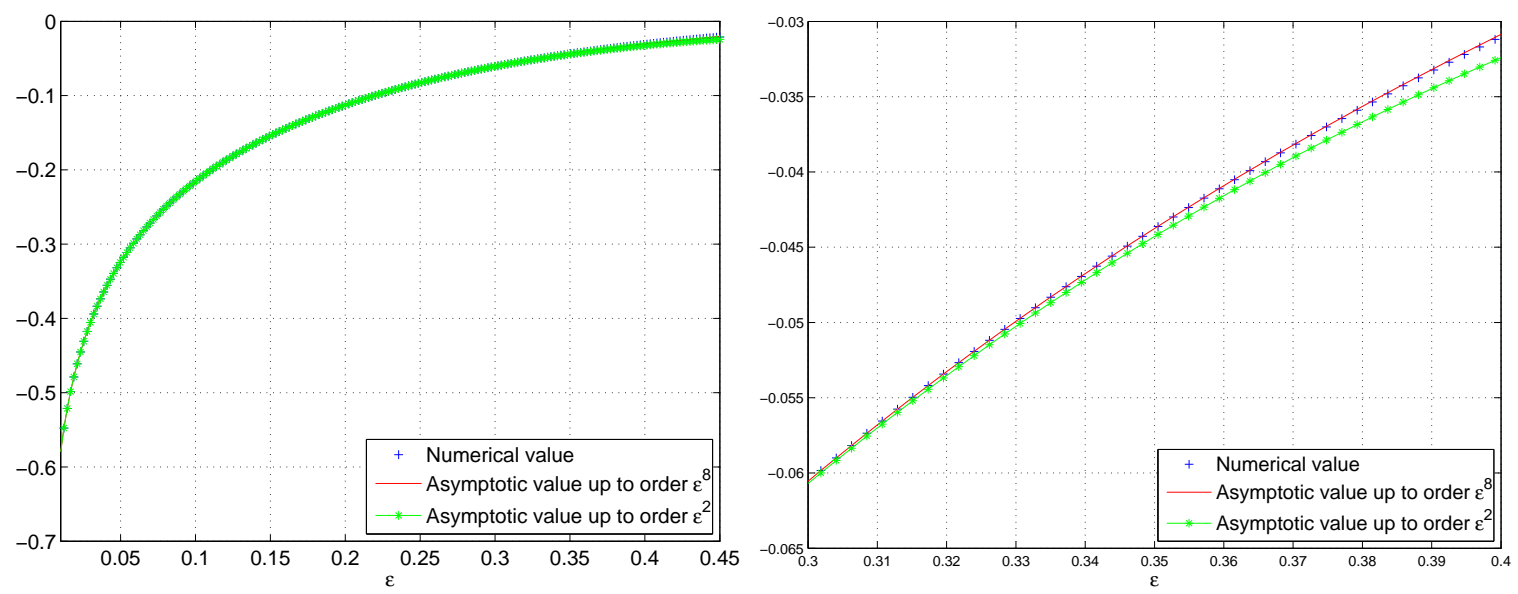

FiguRE 3. Value of the maximum voltage drop using formula (4.4) with and without the term of order $O\left(\epsilon^{8}\right)$ and comparison with the value computed numerically. At the right hand side the maximum has been plotted for values of $\varepsilon \in(0.3,0.4)$ to show the differences between the asymptotic formulae with and without the order $\varepsilon^{8}$ term.

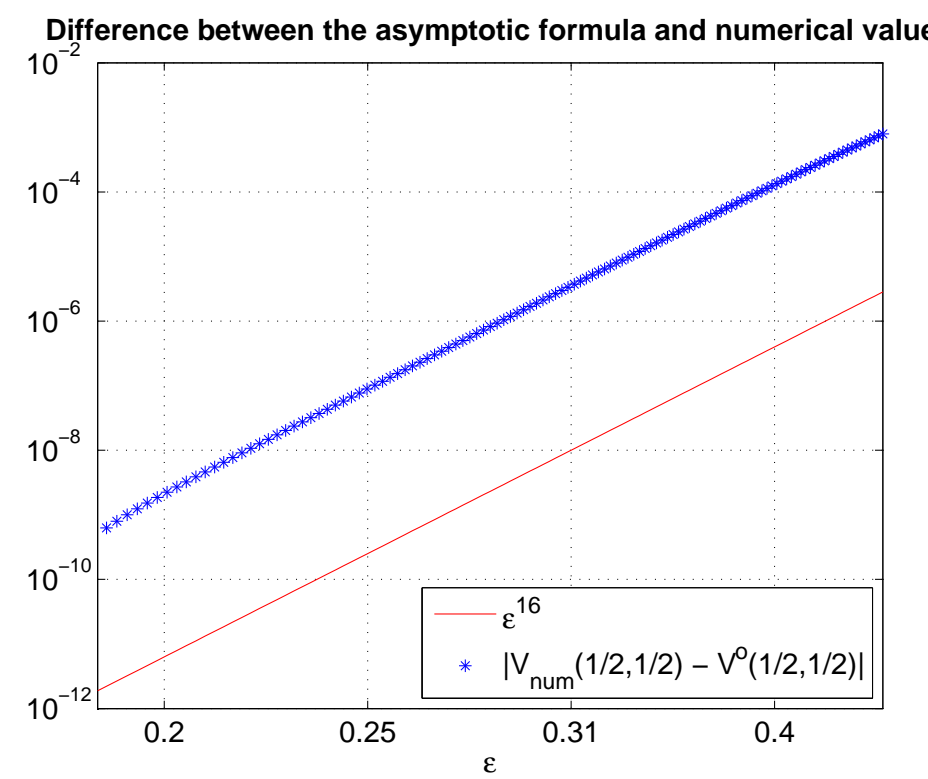

FiguRE 4. Error between formula (4.4) and the numerical value in a logarithmic scale in both axes. The error is indeed parallel to $\varepsilon^{16}$. 


\section{Proof of the Lemma AND CONCLUding REMARKS}

We first note that $v_{1}(1 / 2,1 / 2)=-1 / 12$, because $\sum_{n=1}^{\infty} \frac{(-1)^{n+1}}{n^{2}}=\pi^{2} / 12$. Also, the value of

$$
v_{2}(1 / 2,1 / 2)=\sum_{n, m=1}^{\infty} \frac{(-1)^{n+m}}{\pi^{2}\left(n^{2}+m^{2}\right)}
$$

is $1 / 12-(\log 2) /(4 \pi)$. This is one of the so-called Madelung constants (see [7]). So, we obtain (3.14).

The function $v_{1}$ in (3.10) is easy to identify, since the cosine series $\sum_{\ell=1}^{\infty} \cos (2 \pi \ell t) /\left(2 \pi^{2} \ell^{2}\right)$ corresponds to the function $\frac{1}{2}\left(t-\frac{1}{2}\right)^{2}-\frac{1}{24}$ for $0<t<1$, extended as an even and 2-periodic function outside $[0,1]$. So, for $0<x, y<1$ we have

$$
v_{1}(x, y)=\frac{1}{6}-\frac{1}{2}(\mathfrak{R e} z+\mathfrak{I m} z)+\frac{1}{2}|z|^{2} .
$$

Let us now study $v_{2}(x, y)$ in (3.10) $)$. We define

$$
F_{m}(x, y):=\sum_{n=1}^{\infty} \frac{\cos (2 \pi n x) \cos (2 \pi m y)}{\pi^{2}\left(n^{2}+m^{2}\right)},
$$

and using [8] (formula 1445) we have the following exact value

$$
F_{m}(x, y)=\frac{1}{\pi m} \frac{e^{-2 m \pi}}{1-e^{-2 m \pi}} \mathfrak{R e} \cosh (2 \pi m z)+\frac{1}{2 m \pi} \mathfrak{R e} e^{-2 \pi m z}-\frac{\cos (2 \pi m y)}{2 \pi^{2} m^{2}} .
$$

This expression is the sum of three terms. Let us sum them up (with respect to $m$ ) by summing up first the third term, then the second and finally the first. The third is very easy since, as above, we have

$$
\sum_{m=1}^{\infty}-\frac{\cos (2 \pi m y)}{2 \pi^{2} m^{2}}=-\frac{1}{12}+\frac{1}{2} \mathfrak{I m} z-\frac{1}{2} \mathfrak{I m}^{2} z
$$

for $0<y<1$.

Next we sum up the second:

$$
\begin{aligned}
\mathfrak{R e}\left(\sum_{m=1}^{\infty} \frac{1}{2 m \pi} e^{-2 \pi m z}\right) & =-\frac{1}{2 \pi} \mathfrak{R e} \log \left(1-e^{-2 \pi z}\right) \\
& =-\frac{1}{2 \pi}\left[\log |2 \pi z|-\mathfrak{R e}(\pi z)+\sum_{n=1}^{\infty} \frac{B_{2 n}}{(2 n) ! 2 n} \mathfrak{R e}(2 \pi z)^{2 n}\right]
\end{aligned}
$$


where $B_{2 n}$ are the Bernoulli numbers. And the first one is given by

$$
\frac{1}{\pi} \sum_{n=0}^{\infty} \frac{1}{(2 n) !} Q_{n} \mathfrak{R e}(2 \pi z)^{2 n},
$$

where $Q_{n}=\sum_{m=1}^{\infty} m^{2 n-1} \frac{e^{-2 m \pi}}{1-e^{-2 m \pi}}$ are Lambert series.

From this we see that we have to calculate the two Lambert series

$$
Q_{0}=\sum_{m=1}^{\infty} \frac{1}{m} \frac{e^{-2 m \pi}}{1-e^{-2 m \pi}}, \quad Q_{1}=\sum_{m=1}^{\infty} m \frac{e^{-2 m \pi}}{1-e^{-2 m \pi}} .
$$

The computations below show that

$$
Q_{0}=-\frac{\pi}{12}+\frac{1}{2} \log (\sqrt{2} / G) \quad \text { and } \quad Q_{1}=\frac{1}{24}-\frac{1}{8 \pi} .
$$

Collecting up all that we already know about $v_{1}$ and $v_{2}$ we get

$$
\begin{aligned}
\left(v_{1}+v_{2}\right)(x, y) & =-\frac{1}{2 \pi} \log |z|+\underbrace{\left(\frac{1}{12}+\frac{Q_{0}}{\pi}-\frac{\log (2 \pi)}{2 \pi}\right)}_{=\frac{1}{2 \pi} \log \left(\frac{\sqrt{2}}{2 \pi G}\right)}+\underbrace{\left(\frac{1}{2}+2 \pi Q_{1}-\frac{\pi}{12}\right)}_{=\frac{1}{4}} x^{2} \\
+ & \underbrace{\left(\frac{1}{2}-2 \pi Q_{1}+\frac{\pi}{12}-\frac{1}{2}\right)}_{=\frac{1}{4}} y^{2}+\sum_{n=1}^{\infty} \gamma_{2 n+2} \Re \mathfrak{e} z^{2 n+2} .
\end{aligned}
$$

The value of $Q_{0}$ comes from the following calculation:

$$
\begin{gathered}
Q_{0}=\sum_{m=1}^{\infty} \frac{1}{m} \frac{e^{-2 m \pi}}{1-e^{-2 m \pi}}=\sum_{m=1}^{\infty} \sum_{k=0}^{\infty} \frac{1}{m} e^{-2 m \pi} e^{-2 m k \pi}=\sum_{k=0}^{\infty} \sum_{m=1}^{\infty} \frac{1}{m} e^{-2 m \pi(k+1)} \\
=-\sum_{k=0}^{\infty} \log \left(1-e^{-2 \pi(k+1)}\right)=-\log \left(\prod_{k=1}^{\infty}\left(1-e^{-2 \pi k}\right)\right)=-\log \left(\frac{\sqrt[4]{\pi} e^{\pi / 12}}{\sqrt{2} \Gamma(3 / 4)}\right) .
\end{gathered}
$$

This infinite product appears in the section of Products Involving Theta Functions, and specific values of the Inverse Elliptic Nome, both in [6]. Finally, using the definition of Gauss' constant and the relation $\Gamma(3 / 4)=\sqrt{2} \pi / \Gamma(1 / 4)$ one gets the stated value of $Q_{0}$.

The simplest way to obtain $Q_{1}$ is to observe that $v_{1}+v_{2}$ must be symmetric in the $(x, y)$ variables, and then from formula (6.2) one has

$$
\frac{1}{2}+2 \pi Q_{1}-\frac{\pi}{12}=\frac{1}{2}-2 \pi Q_{1}+\frac{\pi}{12}-\frac{1}{2}
$$


and thus

$$
Q_{1}=\frac{1}{24}-\frac{1}{8 \pi}
$$

The symmetry of $v_{1}+v_{2}$ also shows that the coefficients $\gamma_{4 n+2}$ with $n=1,2, \ldots$ vanish. To obtain the expression of the coefficients $\gamma_{4 n}$ one has to consider the Weierstrass's elliptic function [1]

$$
\begin{aligned}
\wp(z) \equiv \wp(z ; 1 / 2, i / 2)= & \frac{1}{z^{2}}+\sum_{(m, n) \neq(0,0)}\left[\frac{1}{(z-(m+i n))^{2}}-\frac{1}{(m+i n)^{2}}\right] \\
& =\sum_{(m, n)} \frac{1}{(z-(m+i n))^{2}}=\frac{1}{z^{2}}+\sum_{k=1}^{\infty}(4 k-1) G_{4 k} z^{4 k-2},
\end{aligned}
$$

that may be expressed as follows (page 460 in [19]):

$$
\wp(z)=\pi\left(1-\frac{2 \pi}{3}\right)-\frac{\pi^{2}}{\sinh ^{2}(\pi z)}+8 \pi^{2} \sum_{m=1}^{\infty} m \frac{e^{-2 m \pi}}{1-e^{-2 m \pi}} \cosh (2 \pi m z) .
$$

On the other hand, collecting terms one gets

$v_{1}(x, y)+v_{2}(x, y)=\frac{1}{12}-\frac{1}{2} \mathfrak{R} z+\frac{1}{2} \mathfrak{R e} \mathfrak{e}^{2} z-\frac{1}{2 \pi} \mathfrak{R e} \log \left(1-e^{-2 \pi z}\right)+\frac{1}{\pi} \sum_{m=1}^{\infty} \frac{1}{m} \frac{e^{-2 m \pi}}{1-e^{-2 m \pi}} \mathfrak{R e} \cosh (2 \pi m z)$,

and consequently $D_{x}^{2} V_{1}^{o}(x, y)=\frac{1}{2 \pi} \mathfrak{R e}(\wp(z))+1 / 2$, which yields the following identification between the coefficients $\gamma_{4 n}$ and $G_{4 n}$ :

$$
\gamma_{4 n}=\frac{1}{8 \pi n} G_{4 n}
$$

and it is well-known that the coefficients $G_{4 n}$ may be obtained from the invariant $g_{2}=4 \pi^{4} G^{4}$ through the formula [1]: $G_{4}=g_{2} / 60$ and the recurrence $G_{4 n}=\frac{3}{\left(16 n^{2}-1\right)(2 n-3)} \sum_{m=1}^{n-1}(4 m-$ 1) $(4(n-m)-1) G_{4 m} G_{4(n-m)}$.

Finally, $g_{2}$ has been computed using the formula

$$
g_{2}=\frac{4 \pi^{4}}{3}\left[\theta_{2}^{8}\left(0, e^{-\pi}\right)+\theta_{3}^{8}\left(0, e^{-\pi}\right)-\theta_{2}^{4}\left(0, e^{-\pi}\right) \theta_{3}^{4}\left(0, e^{-\pi}\right)\right]
$$

where $\theta_{2}$ and $\theta_{3}$ are two Jacobi's Theta Functions, whose values are (see [6] for details) $\theta_{2}\left(0, e^{-\pi}\right)=(\pi / 2)^{1 / 4} \Gamma^{-1}(3 / 4)=\sqrt{G}$ and $\theta_{3}\left(0, e^{-\pi}\right)=\pi^{1 / 4} \Gamma^{-1}(3 / 4)=\sqrt{\sqrt{2} G}$. This is what it is stated in the lemma. 
Two remarks are in order: Firstly, from the relation $D_{x}^{2} V_{1}^{o}(x, y)=\frac{1}{2 \pi} \mathfrak{R e}(\wp(z))+1 / 2$, we find the well-known formulae that allow us to compute explicitly the sum of Lambert series $Q_{n}$ (see for example the chapter about modular forms in [11]).

Indeed, the Green's function $V_{1}^{o}(x, y)$ has the following expansion

$$
\begin{aligned}
V_{1}^{o}(x, y)=\quad & C(\epsilon)-\frac{1}{2 \pi} \log |z|+\frac{1}{2 \pi} \log \left(\frac{1}{\sqrt{2} \pi G}\right)+\frac{1}{4}|z|^{2}+\frac{1}{\pi} \sum_{n=2}^{\infty} \frac{1}{(2 n) !} Q_{n} \mathfrak{R e}(2 \pi z)^{2 n} \\
& -\frac{1}{2 \pi} \sum_{n=2}^{\infty} \frac{B_{2 n}}{(2 n) ! 2 n} \mathfrak{R e}(2 \pi z)^{2 n} .
\end{aligned}
$$

Therefore, the number $Q_{2 n}$ may be computed in terms of the invariant $g_{2}$ through the formula

$$
Q_{2 n}=\frac{B_{4 n}}{8 n}\left(1-\frac{G_{4 n}}{2 \zeta(4 n)}\right)
$$

where $\zeta$ is the Riemann zeta function. As for the number $Q_{2 n+1}$, it can also be computed in terms of the other invariant $g_{3}$, which in our case is zero, using the formula

$$
Q_{2 n+1}=\frac{B_{4 n+2}}{8 n+4}
$$

For example, we have computed some of these values:

$$
Q_{1}=\frac{1}{24}-\frac{1}{8 \pi}, \quad Q_{2}=\frac{1}{80}\left[G^{4}-\frac{1}{3}\right], \quad Q_{3}=\frac{1}{504}, \quad Q_{4}=\frac{1}{160}\left[3 G^{8}-\frac{1}{3}\right], \quad Q_{5}=\frac{1}{264} .
$$

Secondly, if one considers a domain with the pads arranged in rectangles, $\Omega_{\varepsilon}^{L}=\{(x, y) \mid(x-$ $k)^{2}+(y-L \ell)^{2}>\varepsilon^{2}$, for all integers $\left.k, \ell\right\}$, one can obtain the expression of the maximum voltage in terms of both invariants of the Weierstrass's elliptic function $\wp(z ; 1 / 2, i L / 2)$, i.e., in terms of [1]

$$
g_{2} \equiv 60 \sum_{(m, n) \neq(0,0)} \frac{1}{(m+i n L)^{4}}=\frac{4 \pi^{4}}{3}\left[\theta_{2}^{8}\left(0, e^{-L \pi}\right)+\theta_{3}^{8}\left(0, e^{-L \pi}\right)-\theta_{2}^{4}\left(0, e^{-L \pi}\right) \theta_{3}^{4}\left(0, e^{-L \pi}\right)\right],
$$

and

$$
\begin{aligned}
g_{3} & \equiv 140 \sum_{(m, n) \neq(0,0)} \frac{1}{(m+i n L)^{6}} \\
& =\pi^{6}\left[\frac{8}{27}\left(\theta_{2}^{12}\left(0, e^{-L \pi}\right)+\theta_{3}^{12}\left(0, e^{-L \pi}\right)\right)-\frac{4}{9}\left(\theta_{2}^{4}\left(0, e^{-L \pi}\right)+\theta_{3}^{4}\left(0, e^{-L \pi}\right)\right) \theta_{2}^{4}\left(0, e^{-L \pi}\right) \theta_{3}^{4}\left(0, e^{-L \pi}\right)\right] .
\end{aligned}
$$


For $L=1, \sqrt{2}, 1 / \sqrt{2}, 2,1 / 2, \sqrt{3}, 1 / \sqrt{3}, 3,1 / 3$, the values of $\theta_{2}$ and $\theta_{3}$ are also well-known (see [6]), so one would obtain analytic results for the corresponding maximum voltage.

\section{Conclusion}

The method of matched asymptotic expansions has been used to rigorously obtain the maximum voltage drop in an array bonding power distribution network of a system-on-chip which has constant current consumption and sheet resistance. In [15], the authors obtained an approximate formula for this voltage which we have improved in two ways: first we have found that their order one term was not exactly correct, and second we have derived a systematic way to compute any number of terms in the expansion. It is obvious that for any practical purposes there is no need to obtain the voltage drop up to order $\varepsilon^{8}$, even if $\varepsilon$ is not that small. However, the novelty of our result in this respect is that we have shown that the error that is made when retaining up to order $\varepsilon^{2}$ is as small as $\varepsilon^{8}$. Another important point of our asymptotic scheme is the fact that we found a way to relate the singularities in the outer with well-known, in the electronic engineering literature, multipole solutions.

In the paper [15] a formula similar to (1.1) is also presented for the case of square pads. We plan to study also this case in a future publication, but we can already anticipate two details. First, that in the equivalent to our formula (1.2) there will also appear powers of $\varepsilon^{4}$ and not only powers of $\varepsilon^{8}$. Second, that the Gauss constant $G$ will appear again but for a new reason, that is due to the fact that, as it is well-known, the circle of the same electrical capacity as the square of diagonal $d$ is precisely the circle of diameter $G d$, or, in other words, the conformal map that sends the exterior of the square to the exterior of the disc has a derivative at infinity that is equal to 1 only if the dimensions are in this precise proportion. In general, the outer expansion is exactly the same when one deals with pads of a different shape, while the inner "sees" the actual shape of the path. However, by applying conformal mapping techniques one can write the inner expansion in terms of the corresponding one for circular pads. 
Acknowledgments: The authors are grateful to Prof. J. S. Chapman and Prof. T. Witelski for critically reading the manuscript and to Maria Bruna for assisting us with the numerical simulations. We would also like to thank the anonymous reviewers for providing useful comments and references.

M. Aguareles, J. Haro and J.S. Morales have been supported in part by grants from the Spanish Government (MTM2008-06349-C03-01), and the Catalan Government (2009SGR345), and J. Rius has been partially supported by the spanish MICINN under project TEC20101834.

\section{Appendix 1: ExpANSION up to $O\left(\varepsilon^{2}\right)$ FOR GENERAL PERIODIC ARRAYS}

As suggested by one of the anonymous referee, in this appendix we show an alternative way to derive the first three terms in the expansion for the maximum voltage drop that may be used to extend our results to other types of periodic arrays with circular pads, at least up to order $O\left(\epsilon^{2}\right)$.

We start by considering the equivalent problem to (3.1),

$$
\left\{\begin{array}{c}
\nabla^{2} V=1 \text { in } \Omega_{\varepsilon} \\
V(x, y)=0 \text { on } \partial \Omega_{\varepsilon}, \\
V(x+1, y)=V(x, y), \quad V_{x}(x+1, y)=V_{x}(x, y), \\
V(x, y+1)=V(x, y), \quad V_{y}(x, y+1)=V_{y}(x, y),
\end{array}\right.
$$

where $\Omega_{\varepsilon}$ is now a square of unitary side with a circular hole at the center, $\mathbf{x}=\left(x_{0}, y_{0}\right)=$ $(1 / 2,1 / 2)$ that represents a pad of radius $\varepsilon$.

Following [13], [17] and references therein, in the outer region far away from the hole, the solution should have an expansion of the form,

$$
V^{o} \sim V_{0}^{o} \log \varepsilon+V_{1}^{o}+o(1)
$$

where $V_{0}^{o}$ is just a constant to be found by matching with the inner. For the inner region, which corresponds to considering Laplace equation at the exterior of a disk of radius $\varepsilon$ with homogeneous Dirichlet boundary condition, we use the inner coordinates $\mathbf{x}_{1}=\left(\mathbf{x}-\mathbf{x}_{0}\right) / \varepsilon$, 
so that, to leading order, the inner solution reads,

$$
V^{i}=A \log \left|\mathbf{x}_{1}\right|+o(1)
$$

where $A$ is an arbitrary constant. Upon writing this last expression in terms of the outer coordinates and comparing with the outer expansion,

$$
V^{i}=-A \log \varepsilon+A \log \left|\mathbf{x}-\mathbf{x}_{0}\right|+o(1) \sim V_{0}^{o} \log \varepsilon+V_{1}^{o}+o(1)=V^{o}
$$

it becomes clear that $A=-V_{0}^{o}$ and also, $V_{1}^{o}$ is the solution of

$$
\left\{\begin{array}{c}
\nabla^{2} V_{1}^{o}=1-2 \pi V_{0}^{o} \delta_{\left(x_{0}, y_{0}\right)} \text { in }[0,1]^{2}, \\
V(x+1, y)=V(x, y), \quad V_{x}(x+1, y)=V_{x}(x, y), \\
V(x, y+1)=V(x, y), \quad V_{y}(x, y+1)=V_{y}(x, y) .
\end{array}\right.
$$

The divergence theorem readily gives that $V_{0}^{o}=1 /(2 \pi)$ in order for this equation to have a solution. Furthermore, $V_{1}^{o}=G\left(\mathbf{x} ; \mathbf{x}_{0}\right)+\chi$, being $G\left(\mathbf{x} ; \mathbf{x}_{0}\right)$ the periodic Green's function that satisfies (7.2), and $\chi$ is a constant. This Green's function, which is singular as $\mathbf{x} \rightarrow \mathbf{x}_{0}$, has the local expansion,

$$
G\left(\mathbf{x} ; \mathbf{x}_{0}\right) \sim-\frac{1}{2 \pi} \log \left|\mathbf{x}-\mathbf{x}_{0}\right|+R+\frac{\left|\mathbf{x}-\mathbf{x}_{0}\right|}{4}, \quad \text { as } \mathbf{x} \rightarrow \mathbf{x}_{0}
$$

where $R$ is just a constant that is the regular part of the Green's function. Therefore, $V_{1}^{o} \sim$ $-1 /(2 \pi) \log \left|\mathbf{x}-\mathbf{x}_{0}\right|+R+\chi+\left(\left|\mathbf{x}-\mathbf{x}_{0}\right|\right) / 4$, so the matching condition $V_{1}^{o}+1 /(2 \pi) \log \left|\mathbf{x}-\mathbf{x}_{0}\right|=$ $o$ (1) yields $\chi=-R$.

Continuing to the next order in $\varepsilon$, we find that the inner equation for $V_{1}^{i}$ reads,

$$
\left\{\begin{array}{c}
\nabla^{2} V_{1}^{i}=1 \quad \text { in }\left|\mathbf{x}_{1}\right|>1 \\
V_{1}^{i}=0 \quad \text { in }\left|\mathbf{x}_{1}\right|=1 \\
V_{1}^{i} \sim \frac{\left|\mathbf{x}_{1}\right|^{2}}{4} \quad \text { as } \mathbf{x}_{1} \rightarrow \infty
\end{array}\right.
$$

where $V_{1}^{i}$ is actually the correction term in the inner expansion that is of order $\varepsilon^{2}$. It is clear that $V_{1}^{i}=\left(\left|\mathbf{x}_{1}\right|^{2}-1\right) / 4$, so in terms of the outer variables the inner solution reads $V^{i} \sim-1 /(2 \pi) \log \left(\left|\mathbf{x}-\mathbf{x}_{0}\right| / \varepsilon\right)+\left|\mathbf{x}-\mathbf{x}_{0}\right|^{2} / 4-\varepsilon^{2} / 4$. This last expression produces an order $\varepsilon^{2}$ constant that is easily matched with the outer solution by simply adding a constant term in 
the order $\varepsilon^{2}$ outer solution. This finally yields the three-term expansion in the outer region and in particular the value of the maximum, that is given by,

$$
V_{\text {max }} \sim \frac{1}{2 \pi} \log \varepsilon+G\left(0 ; \mathbf{x}_{0}\right)-R-\frac{\varepsilon^{2}}{4}+\ldots
$$

Finally one should compute $R$ and evaluate the Green's function at the origin, which in the particular case where $\Omega=[0,1]^{2}$ yield, as it is shown in the lemma in Section 3, the first three terms in our formula (4.4).

We note that expression (7.4) is valid not only for pads arranged in squared lattices, but would also hold for any other periodic configuration of pads, like hexagonal lattices, which have been considered, for instance, in [17].

\section{REFERENCES}

[1] M. Abramowitz and I. A. Stegun, Handbook of Mathematical Functions, Dover Publications, New York, (1972).

[2] M. Aguareles, J. Blasco, M. Pellicer and J. Solà-Morales, "Voltage Drop in On-Chip Power Distribution Networks", in Grups d'Estudi de Matemàtica i Tecnologia, A. Alabert, J. Saludes and J. Solà-Morales, eds. Centre de Recerca Matemàtica, Bellaterra, Barcelona, Spain (2009).

[3] L. A. Arledge Jr. and W. T. Lynch, "Scaling and Performance Implications for Power Supply and Other Signal Routing Constraints Imposed by I/O Pad Limitations", Proceedings of the IEEE Symposium on IC/Package Design Intergration, (1998), pp. 45-50.

[4] Bender, C.M. and S.A. Orszag, Advanced mathematical methods for scientists and engineers: Asymptotic Methods and Perturbation Theory. Springer (1999).

[5] J.J. Clement, "Electromigration Modeling for Integrated Circuit Interconnect Reliability Analysis", IEEE Transactions on Device and Materials Reliability, vol. 1, no. 1, pp. 33-42 (2001).

[6] A. Dieckmann, Collection of Infinite Products and Series, Physikalisches Institut der Universitat Bonn ( http://pi.physik.uni-bonn.de/ dieckman/InfProd/InfProd.html ).

[7] R. Finch, Mathematical Constants, Cambridge University Press, Cambridge (2003).

[8] I.S. Gradshteyn and I.M. Ryzhik, Table of Integrals, Series and Products, Academic Press (1965).

[9] E.J. Hinch, Perturbation Methods, Cambridge University Press, (1991).

[10] J. D. Jackson, Classical Electrodynamics, 2nd edition, Wiley, New York (1975).

[11] N. Koblitz Introduction to Elliptic Curves and Modular forms. Springer-Verlag, New York (1984).

[12] T. Kolokolnikov, M.S. Titcombe, M.J. Ward, "Optimizing the fundamental Neumann Eigenvalue for the Laplacian in a Domain with Small Traps". European J. Appl. Math. vol 16, no. 2, 161 â200 (2005). 
[13] S. Pillay, M.J. Ward, A. Pierce, T. Kolokolnikov, "An asymptotic analysis of the mean first passage time for narrow escape problems: Part I: Two-dimensional domains", Multiscale Model. Simul. vol 8, no. 3, $803 \hat{\mathrm{a} 835}$ (2010).

[14] M. Popovich, A.V. Mezhiba, E.G. Friedman, "Power Distribution Networks with On-Chip Decoupling Capacitors", Springer (2008).

[15] K. Shakeri, J.D. Meindl, "Compact Physical IR-Drop Models for Chip/Package Co-Design of Gigascale Integration (GSI)", IEEE Transactions on Electron Devices vol 52, no 6, 1087-1096 (2005).

[16] R. Straube, M. J. Ward, M. Falcke, Reaction rate of small diffusing molecules on a cylindrical membrane, vol. 129, pp 377-405

[17] D.C. Torney, B. Goldstein, "Rates of diffusion-limited reaction in periodic systems", J. Stat. Phys. vol. 49, no. 3/4, pp 725-749 (1987).

[18] M. Van Dyke Perturbation Methods in Fluid Mechanics. Parabolic Press, Stanford, CA (1975).

[19] E.T. Whittaker and G.N. Watson A course of modern analysis. Fourth Edition. Cambridge University Press, Cambridge (1952). 